\title{
Analysis of Artificial Cobweb Structure Used in Power-line Communication
}

\author{
Liang Zhang ${ }^{1}$ Xiaosheng Liu ${ }^{1}$ Yan Zhou ${ }^{1}$ Dianguo $\mathrm{Xu}^{1}$ \\ ${ }^{1}$ (Harbin Institute of Technology, Harbin 150001, China)
}

\begin{abstract}
In order to improve the reliability of power line commnuication, the all terminal reliability of the structure based on artificial cobweb used in low-voltage power line communication (PLC) has been calculated in this paper. The rerouting algorithm is presented based on the structure of cobweb and the high communication survivability of cobweb structure is proved. The comparative simulation results of throughput and data conflict rate prove that the reliability of power line communication networks based on artificial cobweb.
\end{abstract}

Keywords: power-line communication, artificial cobweb, reliability, survivability, effectiveness

\section{Introduction}

Recent years, the State Grid Corporation of China decided to build a novel nationwide power grid based on smart grid technology. Terminal equipment for PLC (power line communication) on LV (low voltage) distribution networks is the most direct means to exchange information with power companies as well as a competitive technical solution for the construction of the smart grid. Therefore, PLC on LV distribution networks now have been attracting new attentions and becoming one of the hot research topics again in China[1]. So far at home and abroad, research on PLC on LV distribution networks is mainly concentrated in the channel noise characteristics and suppression techniques [2], the channel model and estimation [3], OFDM (Orthogonal Frequency Division Multiplexing) performance analysis and its application on PLC [4], impedance matching and coupling circuit design [5], etc. This paper proved the high reliability of communication for the low voltage PLC network based on artificial cobweb network and presents a local routing reconstruction method for LVPLC, and proves the effectiveness of communication based on artificial cobweb. At last, some comparison simulations are performed to prove the high survivability of LVPLC network based on artificial cobweb networking presented in [6].

\section{Network Reliability}

The anti-destroying ability of network is the effect of network topology to the network reliability from the connectivity point. The all-terminal reliability of network is the measurement of the merits of network connectivity. The analysis of allterminal reliability of network structure is to calculate the probability of normal communications between all nodes in accordance with the given constraints.

When the actual network is abstracted as a graph $G$, in order to make better use of mathematical tools, the following three assumptions are also needed [7]: (1) failure probability of the nodes and edges in graph $G$ is known; (2) the nodes and edges have only two status of failure and normal in graph $G$; (3) the failure proba- 
bility status for the nodes and edges are statistically independent of each other in graph $G$.

\subsection{Factorization method}

The factorization method can be expressed as the equation (1). $G$ is a network topology; $p$ is the probability of successful communication between the two nodes connected by any one link $e$ in $G ; q$ is the probability of failure communication of any one link $e$, and $q=1-p$.

$$
R_{A L L}(G, p)=p R(G * e)+q R(G-e)
$$

$R_{A L L}(G, p)$ is the all-terminal reliability of network $G$, i.e., the probability of reliable communication between all nodes; $G * e$ is the network which is produced by merging the two nodes connected by link $e$ in network $G ; G-e$ is the network without link $e$ from network $G$.

To reduce the complexity of the graph $G$, thus to reduce the calculation difficulty of the network reliability based on factorization method, series and parallel simplifying rules are needed to make some protective reduction to graph $G$ [8]. Figure 1(a) shows the series simplifying rules. While there are two series links between source node $u$ and destination node $w$, series simplifying rules are used to merge the two links together, after merging the reliability between link $u$ and link $w$ is $p_{1} \times p_{2}$. Figure 1(b) shows the parallel simplifying rules. While there are two parallel links between source node $u$ and destination node $v$, parallel simplifying rules are applied to merge the two links, and after merging the reliability between link $u$ and link $v$ is $1-q_{1} * q_{2}$.

The LV distribution network is a tree network $[9,10]$, hence the analysis of allterminal reliability of such network is targeted. To ensure the fairness analysis of all-terminal reliability of different net- works based on factorization method, networks with same number of nodes are used to make comparative analysis. For simple calculation, this paper utilizes a variety of typical networks of 7 nodes, which can represent their network characteristics shown in Figure2. The allterminal reliability of the tree, ring, cobweb network are shown as equation (2), (3), (4) respectively.

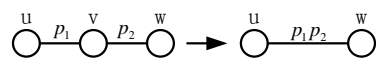

(a) Series simplifying rule

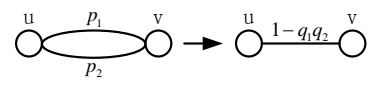

(b) Parallel simplifying rule

Fig.1: Rules of series and parallel
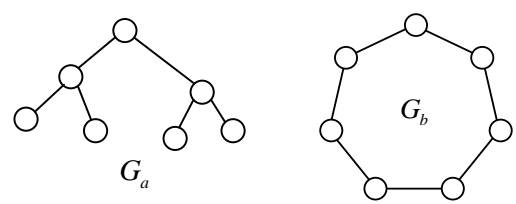

(a) Tree network

(b) Single ring network

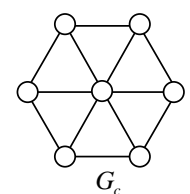

(c) Single layer cobweb

Fig.2: Schemes of factoring theorem

$$
\begin{aligned}
& R_{A L L}\left(G_{a}, p_{g}\right)=p_{g}{ }^{6} \\
& R_{A L L}\left(G_{b}, p_{g}\right)=5 p_{g}{ }^{6}-4 p_{g}{ }^{7} \\
& R_{A L L}\left(G_{c}, p_{g}\right)=51 p_{g}{ }^{12}-394 p_{g}{ }^{11}+1282 p_{g}{ }^{10} \\
& -2256 p_{g}{ }^{9}+2272 p_{g}{ }^{8}-1246 p_{g}{ }^{7}+292 p_{g}{ }^{6}
\end{aligned}
$$

Figure 3 shows the contrast calculation results of all-terminal reliability of tree, single-layer ring and single-layer cobweb network. The horizontal axis is the probability value $p$ of link state, while the ver- 
tical axis is the all-terminal reliability of network. It can be clearly seen from the figure that: in the same link state, the allterminal reliability of single-layer cobweb is higher than that of other kinds of network;

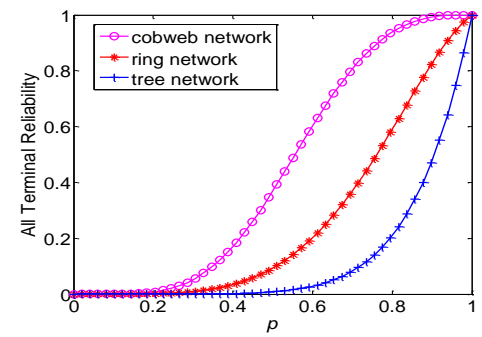

Fig.3: Calculative of all terminal reliability

\section{2 Analysis of Survivability}

The survivability of network reflects the reliability of the communication network in the random destruction. For random "blind nodes" (fault node which cannot communicate with BS caused by the change of link environment) in the actual LVPLC network, combined with the special structure of single-layer artificial cobweb, from the aspect of route maintenance, a routing reconstruction algorithm is proposed. In the meanwhile, routing reconstruction for node $r$ in sub-network is needed to solve the problem of blind node shown in Figure4. In the process of routing reconstruction, the physical signal strength of receipt information is recorded by all nodes. Rerouting reconstruction steps are listed as follows:

(1) A reconstruction broadcast is sent by the central node $n$ to all nodes in the same sub-network.

(2) Nodes that have monitored the reconstruction instructions will transmit the instructions in turns, and add the logical ID in the data packet.

(3) Node $r$ is responsible for recording the signal physical strength of the reconstruction broadcast to calculate and select the node of maximum signal strength composite index $\beta$ (this paper sets the node as $\mathrm{p}$ ) as a relay node. The response message is sent to node $p$, node $r$ with the relay node $\mathrm{p}$ reestablishes a new communication route with central node $\mathrm{n}$. $\beta=s \times \eta^{l}$, here $s$ is the characterization value of the physical signal strength, $l$ is the subnet level of message source, $\eta$ is the success rate of communication between different subnets. The value of $\eta$ can be acquired by the statistical result of the actual experiment. The higher of $\eta$, the higher of the reliability between different levels, on the contrary, the lower of the reliability.

(4) Node $p$ sends a message to node n, and node $\mathrm{n}$ updates the routing tables.

(5) Node $n$ notifies upwards level by level the corresponding nodes of route to update routing tables, until to the gateway. Up to this point, the routing reconstruction process of blind nodes is completed.

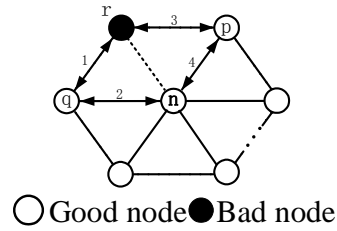

Fig.4: Schematic of routing reconstruction

\section{Simulation results}

\subsection{Simulation environment and pa- rameters}

According to the actual LV distribution environment, this paper selects a $50 \mathrm{~m}$ radius area with 14 user terminals and a BS node. PC is the simulation hardware platform and software Opnet14.5 is the simulation environment. Simulation model of 2 subnets can represent the corresponding network features after networking. The network topology after networking is shown in Figure5. Subnet_0 is the base station node, while subnet_1 and subnet_2 are central nodes of its respective subnet, the rest are terminal nodes. Ac- 
cording to the Konnex standard [11], the command data size of narrowband PLC is 1-15 bits, the corresponding data packet size should be less than 25 bits, actually the data packet size in this paper is 24 bits.

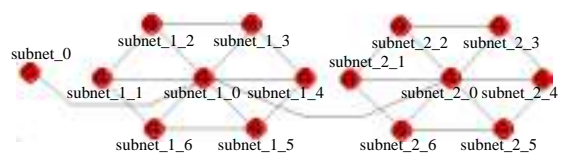

Fig.5: Simulation model

\subsection{Results and analysis of simulation}

After the initialization networking for the network, this paper takes the node subnet_2_2 for instance to confirm the effectiveness of routing reconstruction algorithm. When $p=1$, node subnet_2_2 makes point-to-point communications with node subnet_2_0 through link subnet_2_2-subnet_2_0. Assuming that at the simulation time of $1200 \mathrm{~s}$, node subnet_2_2 turns to be a blind node for $p<1$. Supposing that after routing reconstruction, node subnet_2_2 communicates with central node subnet_2_0 through the relay node subnet_2_1. Figure 6(a) shows the simulation result of the throughput of link subnet_2_2-subnet_2_1 before and after routing reconstruction. While node subnet_2_2 operates normally, this link has no data throughput, after routing reconstruction at the time of $1200 \mathrm{~s}$, throughput of the link increases sharply and eventually stabilizes at 24 bits/s. And it is agreed with the throughput generated by node subnet_2_2, so that it reveals that this link undertakes the throughput of subnet_2_2 after routing reconstruction. Similarly, figure 6(b) shows that, link subnet_2_1-subnet_2_0 undertakes the throughput of subnet_2_2 so that its throughput increases sharply after 1200s and eventually stabilizes at about 50 $\mathrm{bits} / \mathrm{s}$, the sum of throughput of node subnet_2_2 and subnet_2_1. Figure 6(c) shows the simulation result of throughput of link subnet_2_0 - subnet_1_0 before and after routing reconstruction. It is clear from the figure that the throughput of this link is approximately constant after routing reconstruction. And it demonstrates that this routing reconstruction method can well solve the problem of blind node, it improves the survivability of the system and it is somehow practically operational.

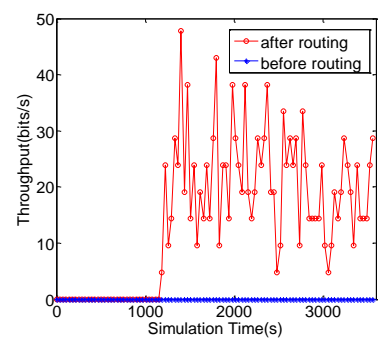

(a)Throughput of link subnet_2_2-subnet_2_1

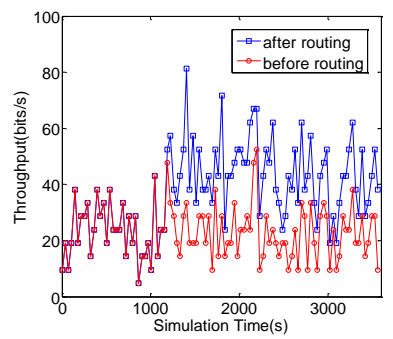

(b)Throughput of link subnet_2_1-subnet_2_0

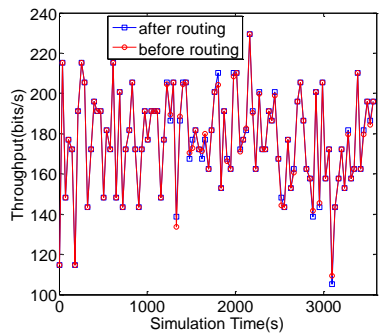

(c)Throughput of link subnet_2_0-subnet_1_0

Fig.6: Simulation results

\section{Conclusion}

This paper makes an analysis of the superiority of communication reliability for single-layer cobweb structure to traditional network, finally the following conclusions are received.

(1) The all-terminal reliability of sin- 
gle-layer cobweb structure is higher than that of traditional tree and ring network, the networking structure of single-layer cobweb subnets is superior to the traditional network structure in the aspects of improving the overall reliability and antidestroying ability of PLC system.

(2) With the premise of high reliability of the cobweb network structure, the routing reconstruction algorithm further improves the system reliability and antidestroying ability. It well meets the requirement of PLC service from the point of throughput and delay. And it solves the problem of blind nodes in the local subnet of the network without routing reconstruction of all nodes in the network, so that it improves the effectiveness of the system.

\section{References}

[1] Ming-Yue Zhai. Transmission Characteristics of Low-Voltage Distribution Networks in China Under the Smart Grids Environment [J]. IEEE Transactions on Power Delivery, pp. 173-180, 2011.

[2] Julian Meng. Noise Analysis of Power-Line Communications Using Spread-Spectrum Modulation [J]. IEEE Transactions on Power Delivery, pp.1470-1476, 2007.

[3] Justinian Anatory, M. M. Kissaka, and N. H. Mvungi. Channel Model for Broadband Power-Line Communication[J]. IEEE Transactions on Power Delivery, pp.135-142, 2007.

[4] Andrea M. Tonello, Francesco Pecile. Efficient Architectures for Multiuser FMT Systems and Application to Power Line Communications[J]. IEEE Transactions on Communica tion, pp.1275-1279,2009.

[5] Antonio Pérez, Albert-Miquel Sánchez, Joan-Ramon Regué, Miquel Ribó, Ricard Aquilué, Pablo Rodríguez-Cepeda, Francisco-Javier Pajares. Circuital and Modal Characterization of the Power-Line Network in the PLC Band[J]. IEEE Transactions on Power Delivery, pp.11821189, 2009.

[6] Liu Xiao-sheng, Zhang Liang, Zhou Yan, $\mathrm{Xu}$ Dian-guo. Performance Analysis of Power Line Communication Network Model Based on Spider Web[J]. 2011 IEEE 8th International Conference on Power Electronics ECCE Asia, pp. 953-959, 2011.

[7] R. Kevin Wood. Factoring Algorithms for Computing K-Terminal Network Reliability[J]. IEEE Transactions on Reliability, pp. 369-278, 1986.

[8] Olympia R. Theologou, Jacques G. Carlier. Factoring \& Reductions for Networks with Imperfect Vertices[J]. IEEE Transactions on Reliability, pp. 210-217, 1991.

[9] Stefano Galli, Anna Scaglione, Zhifang Wang. For the Grid and Through the Grid The Role of Power Line Communications in the Smart Grid[J]. Proceedings of the IEEE, pp.998-1027, 2011.

[10]Q. Gao, J. Y. Yu, P. H. J. Chong, P.L.So, E. Gunawan. Solutions for the "Silent Node" Problem in an Automatic Meter Reading System Using Power-Line Communications [J]. IEEE Transactions on Power Delivery, pp.150-156, 2008.

[11] Konnex (KNX) standard: Konnex Assoc. 2002. 